Алгебра и анализ

Том. 16 (2004), вып. 6
St. Petersburg Math. J. Vol. 16 (2005), No. 6, Pages 943-960 S 1061-0022(05)00885-X

Article electronically published on November 17, 2005

\title{
BI-LIPSCHITZ-EQUIVALENT ALEKSANDROV SURFACES, II
}

\author{
YU. BURAGO
}

\begin{abstract}
It is proved that any two homeomorphic closed Aleksandrov surfaces of bounded integral curvature are bi-Lipschitz-equivalent with constant depending only on their Euler number, upper bounds for their diameters and negative integral curvatures, and two positive numbers $\varepsilon$ and $l$ such that each loop of length at most $l$ bounds a disk of positive curvature at most $2 \pi-\varepsilon$.
\end{abstract}

\section{$\S 1$. BASIC DEFINITIONS AND STATEMENTS}

This paper is a sequel to $\mathrm{BeBu}$. We recall that a map $f: X \rightarrow Y$ of a metric space $\left(X, d_{X}\right)$ to a metric space $\left(Y, d_{Y}\right)$ is said to be bi-Lipschitz with constant $L$ (or L-bi-Lipschitz) if for every $x, y \in X$ we have

$$
L^{-1} d_{X}(x, y) \leq d_{Y}(f(x), f(y)) \leq L d_{X}(x, y) .
$$

In this case, the spaces $X, Y$ are said to be bi-Lipschitz-equivalent (with constant $L$ ). In other words, two metric spaces are $L$-bi-Lipschitz-equivalent if and only if the Lipschitz distance $d_{\text {Lip }}(X, Y)$ does not exceed $\log L$.

We assume that the reader is familiar with the basic notions of the theory of twodimensional manifolds of bounded integral curvature, as exposed, e.g., in AZ and Resh.

In what follows, by an Aleksandrov surface we mean a complete two-dimensional manifold of bounded curvature and with boundary; the boundary (which may be empty) is assumed to consist of finitely many curves with finite variation of turn.

Notation. For an Aleksandrov surface $M$ with metric $d$, we denote by $\omega$ its curvature, which is a signed measure. Let $\omega^{+}, \omega^{-}$be the positive and the negative part of the curvature, and let $\Omega=\omega^{+}+\omega^{-}$be the variation of the curvature. In the case of a Riemannian 2-manifold $M$, we have $\omega^{+}(E)=\int_{E} K^{+} d S, \omega^{-}(E)=\int_{E} K^{-} d S$ for any Borel set $E \in M$, where $K$ is the Gaussian curvature.

The interior points $p$ with curvature $2 \pi$ and the boundary points with turn $\pi$ are called peak points.

By $|x y|$ we denote the distance $d(x, y)$, and by $s(\gamma)$ and $S(E)$ the length of a curve $\gamma$ and the area of a set $E$, respectively. We denote by $\mathbf{D}(X, r)$ the disk of radius $r$ centered at $X$.

For positive numbers $D, C, l, \epsilon$ and an integer $\chi$, we denote by $\mathfrak{M}=\mathfrak{M}(\chi, D, C, l, \epsilon)$ the class of closed oriented Aleksandrov surfaces $M$ having Euler number $\chi$ and satisfying the following conditions:

(i) $\operatorname{diam} M \leq D$;

(ii) $\omega^{-}(M) \leq C$;

2000 Mathematics Subject Classification. Primary 53C45.

Key words and phrases. Bi-Lipschitz map, two-dimensional manifold, bounded integral curvature, Aleksandrov surface.

This work was partially supported by grants RFBR 02-01-00090, SS-1914.2003.1, CRDF RM1-2381ST-02, and by the Shapiro Foundation of Pennsylvania State University. 
(iii) if the length of a simple closed curve in $M$ is less than $l$, then the curve bounds a disk $\mathbf{D} \subset M$ such that $\omega^{+}(\mathbf{D}) \leq 2 \pi-\epsilon$.

Condition (iii) implies that $\omega(p) \leq 2 \pi-\epsilon$ at every point $p \in M$. In particular, $M$ has no peak points. Moreover, the systolic constant for $M$ is at least $l$. (Recall that the systolic constant $\operatorname{sys}(M)$ of a closed surface $M$ is the infimum of the lengths of the closed noncontractible curves in $M$. This notion is meaningful only if $\pi_{1}(M)$ is nontrivial.)

The classes $\mathfrak{M}$ are compact; the proof of this is standard; see $₫ 2$.

Here is the main result of the paper.

Theorem 1. There exists a positive constant $L$ depending only on $\chi, D, C, l, \epsilon$ and such that $d_{\text {Lip }}\left(M_{1}, M_{2}\right) \leq L$ for any two Aleksandrov surfaces $M_{1}, M_{2} \in \mathfrak{M}$.

Remark 1. a) Obviously, a similar theorem is true for nonoriented surfaces.

b) Theorem 1 is a generalization of $[\mathrm{BeBu}$, Theorem 1], but the proof of Theorem 1 depends on the latter theorem.

c) Theorem 11 can be generalized to some classes of compact Aleksandrov surfaces with nonempty boundary. (Recall that the boundaries were assumed to consist of finitely many curves with bounded variation of turn.) Naturally, we must add some boundary conditions to describe the classes $\mathfrak{M}^{\prime}$ of surfaces with boundary. For instance, it suffices to consider a class of surfaces with boundary such that their doublings belong to some fixed class $\mathfrak{M}$. In particular, this condition includes the requirements that the distances between the components of the boundary be uniformly bounded away from zero and that the turn of the boundary at every point be less than $\pi-\epsilon^{\prime}$ for some $\epsilon^{\prime}>0$. These requirements are neither necessary nor sufficient. We drop a formulation of the necessary and sufficient conditions because they are somewhat complicated.

In the case of surfaces with boundary, the proof is basically the same as for closed surfaces. If we consider a surface whose doubling belongs to $\mathfrak{M}$, it suffices to apply Theorem 1 to the doubling, because freedom in the choice of a bi-Lipschitz map allows us to find a map that takes boundaries onto boundaries.

Let $T$ be a tube (i.e., an Aleksandrov surface homeomorphic to a closed disk punctured at the center and such that $d\left(a, p_{i}\right) \rightarrow \infty$ as $i \rightarrow \infty$ for any sequence of points $p_{i} \in T$ whose images in the disk converge to its center; here $a$ is a fixed point). The quantity $v(T)=-\tau(\gamma)-\omega(T)$ is called the growth speed of the tube $T$. Here $\tau(\gamma)$ is the turn of the boundary $\gamma$ of the tube. The Cohn-Vossen inequality shows that $v \geq 0$. Note that the growth speed of a tube is positive if and only if

$$
\lim _{i \rightarrow \infty} \frac{l\left(\gamma_{i}\right)}{d\left(a, p_{i}\right)}>0,
$$

where $l\left(\gamma_{i}\right)$ is the length of the shortest noncontractible loop with vertex $p_{i}$. Under the condition $\Omega(T)<\infty$, this limit is well defined and does not exceed 2 .

Every open (i.e., equipped with a complete and unbounded metric) finitely connected Aleksandrov surface can be cut by loops into a compact part $M_{c}$ and tubes $T_{i}$. We consider the classes $\mathfrak{M}^{*}=\mathfrak{M}^{*}\left(g, n, C, l, \epsilon, v_{0}\right)$ of (oriented) Aleksandrov surfaces $M$ of genus $g$ that have $n$ tubes and satisfy the following conditions:

the growth speed of each tube is at least $v_{0}>0$;

conditions (ii) and (iii) are fulfilled for $M$.

Observe that all surfaces of any class $\mathfrak{M}^{*}$ are homeomorphic, and that the growth speed of a tube does not depend on the choice of its boundary. We can choose the tubes $T_{i}$ in such a way that their boundaries $\gamma_{i}$ satisfy $\Omega\left(T_{i}\right)+\tau^{+}\left(\gamma_{i}\right)<0.001$, where $\tau$ is the turn from the side of $T_{i}$. Note that condition (iii) is fulfilled for $M_{c}$. To ensure this, 
we take loops sufficiently far from the fixed point of $M$ and make them "as round as possible".

We denote by $\widetilde{\mathfrak{M}}=\widetilde{\mathfrak{M}}\left(g, n, D, C, l, \epsilon, s, v_{0}\right)$ the subset of $\mathfrak{M}^{*}$ formed by the surfaces that can be decomposed into a compact part $M_{c}$ and tubes $T_{i}$ satisfying the previous conditions and the additional conditions

$$
\operatorname{diam} M_{c} \leq D, \quad \operatorname{length}\left(\gamma_{i}\right) \leq s .
$$

It is clear that every surface of class $\mathfrak{M}^{*}$ belongs to some class $\widetilde{\mathfrak{M}}$. The corollary below is a consequence of Theorem 1 and Remark 2 in $\mathrm{BeBu}$.

Corollary 1. For any $g, n, D, C, l, \epsilon, s$, and $v_{0}$, there exists a constant $L_{1}$ depending only on these numbers and such that all Aleksandrov surfaces that belong to the class $\tilde{\mathfrak{M}}\left(g, n, D, C, l, \epsilon, s, v_{0}\right)$ are $L_{1}$-bi-Lipschitz-equivalent.

The author thanks A. Belen'kiı and V. Zalgaller whose advice helped to simplify some proofs.

Sketch of the proof of Theorem 1, Let $L(M, N)$ denote the infimum of the Lipschitz constants for the bi-Lipschitz maps $M \rightarrow N$, where $M, N \in \mathfrak{M}(D, C, \chi, l, \epsilon)$. Suppose the theorem fails. This means that there exists a sequence of surfaces $M_{i} \in \mathfrak{M}(D, C, \chi, l, \epsilon)$ such that $L\left(M_{i}, N\right) \rightarrow \infty$, where $N$ is a smooth surface of the same class. (Later we show that we can assume the surfaces $M_{i}$ to be equipped with polyhedral metrics.)

Lemma 2 implies the existence of a subsequence of $\left\{M_{i}\right\}$ convergent in the GromovHausdorff topology and such that the limit space $M$ of this subsequence is an Aleksandrov space of the same class $\mathfrak{M}(\chi, D, C, l, \epsilon)$. In particular, $\omega(p) \leq 2 \pi-\epsilon$ for every point $p \in M$. We may assume that our initial sequence has these properties.

Then from $[\mathrm{BeBu}$, Theorem 1] it follows that $L(M, N)<\infty$. Therefore, we shall arrive at a contradiction if we prove the following lemma.

Lemma 1 (Key Lemma). Under the assumptions made above, for sufficiently large $i$ we have $L\left(M_{i}, M\right) \leq A<\infty$, where $A$ is a constant independent of $i$.

The proof of this lemma constitutes the main part of the paper. The proof itself is presented in $\$ 5$ it is based on some special triangulations of the surfaces $M$ and $M_{i}$ (see \$4) and on the main construction of the paper BL, 1] Auxiliary statements about triangles in $\mathbb{R}^{2}$ and Aleksandrov surfaces are collected in $\$ 3$.

\section{$\S 2$. THE SPACE $\mathfrak{M}$ IS COMPACT}

Lemma 2. The space $\mathfrak{M}=\mathfrak{M}(\chi, D, C, l, \epsilon)$ is compact in the Gromov-Hausdorff topology.

Proof. 1. The fact that the space $\mathfrak{M}$ is precompact was proved in [Sh]; here we present a short proof for completeness. We shall denote by $C^{*}$ various constants depending only on the parameters of the class $\mathfrak{M}$. It suffices to show that for any (sufficiently small) $r>0$, every surface $M \in \mathfrak{M}$ contains an $r$-net of at most $C^{*} r^{-2}$ points.

We fix $r<\frac{1}{4} l$ and consider a maximal $2 r$-separated set $\left\{a_{1}, \ldots, a_{k}\right\}$ of points of the surface $M$. These points form a $4 r$-net. Let $\mathbf{D}$ denote the ball $\mathbf{D}(a, r)$ with $a=a_{i}$, and let $r_{0}$ be the supremum of the numbers $\rho \leq r$ such that the disk $\mathbf{D}\left(a, \rho^{\prime}\right)$ is simply connected for all $\rho^{\prime} \leq \rho$.

\footnotetext{
${ }^{1}$ Added in proof. Now we can simplify the proof by using, instead of the Bonk-Lang method, the global Chebyshev coordinates with bifurcations; the existence of such coordinates has recently been proved by S. Malev and the author (see arXiv:math.DG/0506580).
} 
If $r_{0} \geq \frac{1}{2} r \sin \frac{\epsilon}{2}$, then $S(\mathbf{D}) \geq \frac{1}{8} \epsilon r^{2}\left(\sin \frac{\epsilon}{2}\right)^{2}$. Since the total area of $M$ does not exceed $\left(2 \pi+\omega^{-}(M)\right) \operatorname{diam}^{2} M \leq D^{2}(2 \pi+C)$, the number of such disks does not exceed $C^{*} r^{-2}$.

Now suppose that $r_{0} \leq \frac{1}{2} r \sin \frac{\epsilon}{2}$. Then in $\mathbf{D}\left(a, r_{0}\right)$ there is a geodesic loop $\gamma$ of length $2 r_{0}$ centered at $a$ and separating two components of the boundary of the disk $\mathbf{D}\left(a, r_{0}\right)$. Since $2 r<l$, at least one of the components of $M \backslash \gamma$ is simply connected. Let $K$ denote the closure of this component. The Gauss-Bonnet formula says that $\omega^{+}(K) \geq \pi$. A well-known inequality for the length of a curve in a simply connected region (see, e.g., Resh, 8.5]) yields

$$
R(K) \leq \frac{2 r_{0}}{\sin \frac{\omega^{+}(K)}{2}} \leq \frac{2 r_{0}}{\sin \frac{\epsilon}{2}} \leq r,
$$

where $R(K)$ is the inradius of $K$, i.e.,

$$
R(K)=\sup \{d(x, \partial K), x \in K\} .
$$

This means that $K$ does not intersect the disks $\mathbf{D}\left(a_{j}, r\right)$ with $j \neq i$. Moreover, $\omega^{+}(K) \geq$ $\pi$. Adding the set $K$ to the disk $\mathbf{D}$, we obtain a set that does not intersect the other disks and has curvature at least $\pi$. We perform this procedure for every disk whose radius satisfies $r_{0} \geq \frac{1}{2} r \sin \frac{\epsilon}{2}$, obtaining a family of disjoint sets containing the disks. The positive curvature of each of these sets different from a disk is at least $\pi$. Therefore, the number of such sets and the number of all the disks is at most $C^{*} r^{-2}$.

2. It remains to prove that if $M_{i} \in \mathfrak{M}$ and $M_{i} \rightarrow M$, then $M \in \mathfrak{M}$. In $\underline{\mathrm{Sh}}$, it was proved that the limit space $M$ looks like a graph (possibly, infinite) some vertices of which "are blown up" to certain Aleksandrov surfaces that can be attached to each other only at singletons; see the details in $[\mathrm{Sh}$. Therefore, it suffices to prove that no point $p \in M$ can split its neighborhood $U$. After that, it becomes clear that $M \in \mathfrak{M}$. Indeed, obviously, diam $M \leq D$. Since the curvatures $\omega_{i}$ of the surfaces $M_{i}$ converge weakly (in the sense of Fukaya's definition; see Sh ) to the curvature $\omega$ of $M$, we have $\omega^{-}(M) \leq C$. Now it is easy to check that condition (iii) of the definition of the class $\mathfrak{M}$ is fulfilled for $M$.

So, we prove that no point $p \in M$ can split its neighborhood. Assume the contrary: there is a point $p \in M$ that splits a neighborhood of it. Then it splits any smaller neighborhood of it. Let $U=\mathbf{D}(p, 10 r) \supset \mathbf{D}(p, \rho)=\mathbf{D}$ be split by $p$. We fix $a, b$ in different components of $U \backslash p$, both at distance $r$ from $p$, and choose points $p_{i}, a_{i}$, $b_{i} \in M_{i}$ such that $p_{i} \underset{\mathrm{GH}}{\longrightarrow} p, a_{i} \underset{\mathrm{GH}}{\longrightarrow} a, b_{i} \underset{\mathrm{GH}}{\longrightarrow} b$ (we mean convergence with respect to the Gromov-Hausdorff topology). For all sufficiently large $i$, the distances $\left|a_{i} p\right|,\left|b_{i} p\right|$ are almost equal to $r$.

Now consider the disks $U_{i}=\mathbf{D}\left(p_{i}, 10 r\right)$ and $\mathbf{D}_{i}=\mathbf{D}\left(p_{i}, \rho\right)$, where $\rho \ll r$ (for instance, $\left.\rho<\frac{1}{100}(2 \pi+C)^{-1} r\right)$ and $r<\frac{1}{3} l$. Observe that the length of the boundary of the disk $\mathbf{D}_{i}=\mathbf{D}\left(p_{i}, \rho\right)$ does not exceed $(2 \pi+C) \rho$. Since $r<\frac{1}{3} l$, each of the closed disks $\bar{U}_{i}, \overline{\mathbf{D}}_{i}$ is homeomorphic to a Euclidean closed disk with at least countable union of disjoint open disks removed.

Two cases are possible.

a) For some subsequence of indices $i$, the points $a_{i}$ and $b_{i}$ are located in one component of $U_{i} \backslash \overline{\mathbf{D}}_{i}$. In this case, the points $a_{i}, b_{i}$ can be connected in $U_{i} \backslash \overline{\mathbf{D}}_{i}$ by a path of length at most $3 r+(2 \pi+C) \rho<4 r$. We replace this path by a dotted line consisting of at most $40 r \rho^{-1}$ points and with steps at most $\frac{1}{10} \rho$. Passing to the limit, we get a dotted line "connecting" $a$ and $b$ in $M$ and such that its steps are as small as before. For at least one of the points of the resulting dotted line, the distance of it from $p$ does not exceed $\frac{1}{10} \rho$. This contradicts the fact that the distances between the points $p_{i}$ and the points of the corresponding dotted lines cannot be less than $\rho$. 
b) Suppose the points $a_{i}$ and $b_{i}$ lie in different components of the set $U_{i} \backslash \overline{\mathbf{D}}_{i}$ (for some subsequence). In particular, the closed disks $\overline{\mathbf{D}}_{i}$ are not simply connected. Then in $\overline{\mathbf{D}}_{i}$ there is a simple closed loop of length at most $3 \rho$ and such that it separates the components of the points $a_{i}, b_{i}$. This loop is contractible because $3 \rho<l$. Therefore, the loop bounds a disk $\mathbf{D}^{\prime}$ containing one of the components; we assume that this is the component of $a_{i}$. The Gauss-Bonnet theorem implies that $\omega^{+}\left(\mathbf{D}^{\prime}\right) \geq \pi$. If we choose $\rho<\frac{1}{100} \sin \frac{\epsilon}{2}(2 \pi+C)^{-1} r$, then the distance from $a_{i}$ to the boundary of $\mathbf{D}^{\prime}$ does not exceed

$$
\frac{\text { length of } \partial \mathbf{D}^{\prime}}{\sin \frac{\epsilon}{2}} \leq 3 \rho(2 \pi+C)\left(\sin \frac{\epsilon}{2}\right)^{-1} \leq \frac{3}{100} r .
$$

Consequently, the distances between $a_{i}$ and the disks $\mathbf{D}_{i}$ do not exceed $\frac{3}{100} r$. Thus, $\left|p a_{i}\right| \leq \rho+\frac{3}{100} r<\frac{1}{2} r$, a contradiction.

The lemma is proved.

\section{§3. LEMmas ABOUT TRIANGLES}

Here we collect some auxiliary statements about triangles on Aleksandrov surfaces. These lemmas will be used in $\S \S 5,6$. Basically, we deal with modifications of statements proved in $\mathrm{BeBu}$, and $\mathrm{AZ}$.

Along with usual triangles, we sometimes consider generalized triangles. By a generalized triangle we mean a disk bounded by three polygonal lines (sides of the triangle) whose edges are minimizers (i.e., shortest paths). We assume that the lengths of these sides satisfy the strict triangle inequality. The sum of the absolute curvature of a generalized triangle $T=\triangle A B C$ and the variations of turn of its sides will be called the total curvature of $T$ and will be denoted by $\widetilde{\Omega}(T)$, i.e., $\widetilde{\Omega}(T)=\Omega(T)+\sigma(A B)+\sigma(B C)+\sigma(C A)$, where $\sigma$ means the variation of turn from the triangle side. The angles of a generalized triangle can be zero. For brevity, we sometimes drop the word "generalized".

Recall that, on an Aleksandrov surface, a comparison triangle for a (generalized) triangle $T$ is a planar triangle with the same side lengths.

Usually, we consider generalized triangles $T$ with small $\widetilde{\Omega}(T)$. If this quantity is small as compared to the angles of $T$, then $T$ is bi-Lipschitz-equivalent to its comparison triangle, with Lipschitz constant depending on the lower bound for the angles. More precisely, the following statement is true.

Lemma 3. For any $\alpha>0$ and $L>1$, there exists $\delta=\delta(\alpha, L)>0$ with the following property. If every angle of a generalized triangle $\triangle A B C$ is at least $\alpha$ and $\widetilde{\Omega}(\triangle A B C)<$ $\delta$, then there exists an L-bi-Lipschitz map of the generalized triangle $\triangle A B C$ onto its comparison triangle. This map can be chosen so that its restriction to the boundary of the triangle is an isometry taking vertices to vertices.

This lemma is a minor modification of Lemma 4 in $[\mathrm{BeBu}$ and can be proved the same way. For this reason, we omit the proof. We also need a more general statement.

Lemma 4. Consider a simply connected closed region $T$ equipped with a polyhedral metric. Suppose $T$ is bounded by two minimizers $B A, B C$ and a polygonal line $A C$. Assume that $|B A|+|B C|>s(A C)$, where $s(A C)$ is the length of $A C$.

Suppose that $T$ is starlike with respect to a point $B$, i.e., all minimizers $B X$, where $X \in$ $A C$, intersect $A C$ only at $X$. We assume that the angle $\angle A B C$ satisfies the condition $0<\phi \leq \angle A B C \leq \frac{1}{10}$ and that for every $X \in A C$ the angle between an arbitrary minimizer $B X$ and an arbitrary arc emanating from $X$ and pertaining to the polygonal line $A C$ is in the interval $\left[\frac{\pi}{2}-\frac{1}{10}, \frac{\pi}{2}+\frac{1}{10}\right]$. 
Then there exist constants $\delta>0$ and $L \geq 1$ such that if $\widetilde{\Omega}(T)<\delta$, then $T$ is $L$-biLipschitz-equivalent to a planar triangle $\triangle A^{\prime} B^{\prime} C^{\prime}$ with side lengths $|A B|,|C B|, s(A C)$, respectively.

Moreover, if $A C$ is a minimizer, then $L$ can be chosen as a function $L=L(\delta)$ in such a way that $L \rightarrow 1$ as $\delta \rightarrow 0$.

Recall that it is assumed that an $L$-bi-Lipschitz map of $T$ onto its "comparison triangle" $A^{\prime} B^{\prime} C^{\prime}$ preserves the lengths of the boundary curves.

Again, this lemma is a modification of Lemma 4 in $\mathrm{BeBu}$ and can be proved in the same way, so that we omit the details, presenting only the outline of the proof. First, we map $T$ onto a closed planar region $\widetilde{T}$ bounded by intervals $A_{1} B_{1}, C_{1} B_{1}$ and a polygonal line $A_{1} C_{1}$ such that $\left|A_{1} B_{1}\right|=|A B|,\left|C_{1} B_{1}\right|=|C B|, \angle A_{1} B_{1} C_{1}=\angle A B C$. For this, we use Chebyshev coordinates. It can be verified that the turns of the polygonal line $A_{1} C_{1}$ at its vertices are dominated by a certain quantity depending on $\phi$ and on the smallness of $\delta$. After that, it is not difficult to map $\widetilde{T}$ onto the comparison triangle $\triangle A^{\prime} B^{\prime} C^{\prime}$.

We need the following corollary.

Corollary 2. Consider a convex boundary quadrangle $\square=A A_{1} C_{1} C$ bounded by four minimizers. Given $0<\phi, \nu<\frac{1}{10}$, suppose that

$$
\left|A A_{1}\right|=\left|C C_{1}\right|, \angle A_{1}>\frac{\pi}{2}+\nu, \angle C_{1}>\frac{\pi}{2}+\nu,\left|\angle A-\frac{\pi}{2}\right|<\phi,\left|\angle C-\frac{\pi}{2}\right|<\phi .
$$

Then there is a function $L=L(\delta) \geq 1$ such that $L \rightarrow 1$ as $\delta \rightarrow 0$, and if $\widetilde{\Omega}(\square)<\delta$, then $\square$ is L-bi-Lipschitz-equivalent to a planar quadrangle with the same side lengths and such that the differences between its angles $\angle A_{1}^{\prime}, \angle C_{1}^{\prime}$ and the angles $\angle A_{1}, \angle C_{1}$ do not exceed $C^{*} \delta$.

The function $L(\delta)$ depends on $\nu$ and $\phi$.

To prove the corollary, we extract the quadrangle $\square$ from the surface and attach a planar triangle $O A_{1} C_{1}$ to $\square$ along $A_{1} C_{1}$ in such a way that the sides $A_{1} O, C_{1} O$ of the planar triangle are continuations of the quadrangle sides (i.e., they form angles $\pi$ with the minimizers $A_{1} A, C_{1} C$, respectively). This yields a generalized triangle $T=\triangle O A C$ (not necessarily an ordinary triangle, because its sides may fail to be minimizers). It is easy to check that this triangle satisfies the hypothesis of Lemma 4. Application of this lemma gives a bi-Lipschitz map (with a constant depending only on $\phi$ and the smallness of $\delta) f_{0}: G \rightarrow \triangle O^{\prime} A^{\prime} C^{\prime}$, where $\triangle O^{\prime} A^{\prime} C^{\prime}$ is a comparison triangle for $T$, such that the restrictions of $f_{0}$ to the sides are isometries.

In the proof of Lemma 4, the map $f_{0}$ is constructed in two steps. First, we map $T$ onto a planar figure bounded by two intervals (the images of $O A$ and $O C$ ) and the polygonal line $\gamma$ (the image of the minimizer $A C$ ). For this, we use Chebyshev coordinates. As the second step, the polygonal line $\gamma$ is "rectified"; see the details in $\mathrm{BeBu}$.

Since triangle $\triangle O A_{1} C_{1}$ is planar, the first map acts isometrically on it. In particular, the minimizer $A_{1} C_{1}$ is mapped onto an interval $A_{1}^{\prime} C_{1}^{\prime}$ of the same length. Now it is not difficult to straighten up the polygonal line $\gamma$ keeping the interval $A_{1}^{\prime} C_{1}^{\prime}$ fixed. For this, we cut the quadrangle $A^{\prime} A_{1}^{\prime} C_{1}^{\prime} C^{\prime}$ by the diagonal $A_{1}^{\prime} C^{\prime}$ into two triangles, and, leaving the triangle $A_{1}^{\prime} C_{1}^{\prime} C^{\prime}$ fixed, straighten $\gamma$ in the "curved triangle" $\triangle A_{1}^{\prime} C^{\prime} A^{\prime}$ in the same way as was done in $\mathrm{BeBu}$, item 8 of the proof of Lemma 4].

Remark 2. If it is not stipulated otherwise, the words "bi-Lipschitz equivalence" will always mean the existence of a bi-Lipschitz map with constant depending only on the parameters of the class $\mathfrak{M}$. We always assume that if a surface has a boundary, then the restriction of a bi-Lipschitz map to the boundary is linear. For triangles, we assume that vertices are mapped to vertices. 
By definition, the total curvature $\widetilde{\Omega}(G)$ of a subset $G$ of a generalized triangle $T=$ $\triangle A B C$ is equal to the sum of $\Omega(G)$ and the negative turn of the intersection of the triangle sides with $G$ (we mean open sides, without vertices). Recall that for an ordinary triangle the turns of its sides are nonpositive.

By minimizers connecting points of a triangle we mean minimizers in the induced metric.

Lemma 5. For any positive $\Psi, R$, and $\delta$, there exists a number $r>0$ with the following properties. Let a simple triangle $\triangle A B C$ and a point $Z$ on an Aleksandrov surface be such that $\widetilde{\Omega}(\triangle A B C)<\Psi, \widetilde{\Omega}(\triangle A B C \backslash \mathbf{D}(Z, r))<\delta$, and $|A C|<R$. Then:

(i) if $B=Z,|A C|<R$, and $d(B,[A C])>R$, then the differences between the angles $\angle B, \angle C$ of the triangle and the corresponding angles of its comparison triangle $\triangle A^{\prime} B^{\prime} C^{\prime}$ do not exceed $2 \delta$;

(ii) if $A, B \in \mathbf{D}(Z, r)$ and $|C Z| \geq R$, then $\angle A C B-\angle A^{\prime} C^{\prime} B^{\prime} \leq 2 \delta$, where $\angle A^{\prime} C^{\prime} B^{\prime}$ is the angle of the comparison triangle.

Remark 3. If we choose $r$ in such a way that $\angle A^{\prime} C^{\prime} B^{\prime}<\delta$ in (ii), then, obviously, $\angle A C B<3 \delta$.

Here we restrict ourselves to a sketch of the proof, because the technique is the same as in [AZ, Chapter IV, §2]; the reader can find all details in that book. Note that it suffices to prove the lemma for polyhedral metrics only. Actually, we need only this case.

In case (i), the idea of the proof is as follows: suppose that in the triangle $\triangle A B C$ (with polyhedral metric), there are points of positive curvature at a distance less than $r<R / 2$ from $B$. Then these points $X$ can be moved one by one until they are placed at distances $R / 2$ from $B$. To do this, we find a digon containing the point $X$, having positive curvature only at $X$, and bounded by two minimizers with common ends at $B$ and at yet another point $Y$ lying at a distance $R^{\prime}$ from $B$. After that, we remove this digon and glue the minimizers $B Y$ together. As a result, the vertex $X$ vanishes, but additional curvature can arise at the point $Y$. This additional curvature is at most $r / R^{\prime}$ times the curvature of the removed digon if $|B X| \leq r$, and is not greater than the latter curvature if $|B X| \geq r$. This means that, moving all vertices of positive curvature in $\mathbf{D}(B, r)$ to positions at distance at least $R / 2$ from $B$, we increase the positive curvature by a quantity less than $\delta / 2$ if $r<\frac{1}{4} \delta R \Psi^{-1}$.

After we moved all such points $X$, there will be no positive curvature in the $R / 2$ neighborhood of $B$. Now we can do the next step: move all vertices $Z$ of negative curvature to positions at distance at least $R / 2$ from $B$. For this, we glue additional material in a slit that looks like a tree with one vertex and consists of a slit along $B Z$ and several additional slits starting at $Z$. At this step, negative curvature reduces almost in the same proportion as positive curvature had reduced before. As a result, the variation of curvature becomes less than $2 \delta$. The side $A C$ remains to be a minimizer during this process, because $A C$ is sufficiently far from the deformed part of the triangle. The angles $\angle A$ and $\angle C$ also do not change. This proves (i).

In case (ii), the idea of the proof is almost the same: in the first step we remove all vertices of positive curvature from the intersection of the triangle with $\mathbf{D}(Z, r)$. We do this by cutting off digons with vertex $C$. This allows us to eliminate the entire positive curvature. Choosing $r$ as in (i), we can guarantee that the change of the angle $\angle C$ be at most $\delta$. However, the side $A B$ can cease to be a minimizer. In such a case, we replace it by a minimizer (in the induced metric), which is not longer than the side. The curvature can only become less. Applying the angle comparison theorem to the triangle of nonpositive curvature bounded by $A C, B C$, and a new minimizer $A B$, we immediately obtain the required inequality. 


\section{§4. Approximations And triangulations}

Lemma 6. Every compact Aleksandrov surface $M$ (possibly, with boundary) without peak points can be Lipschitz-approximated by surfaces $P_{i}$ equipped with polyhedral metrics. Moreover, the convergence $P_{i} \stackrel{\text { Lip }}{\longrightarrow} M$ can be made regular; the latter means that $\omega_{i}^{ \pm} \stackrel{\text { weak }}{\longrightarrow}$ $\omega^{ \pm}$

This lemma was announced by Yu. Reshetnyak in Resh1] (actually, in a more general form), but the proof has never been published.

Proof. Recall that a triangle is simple if it is boundary-convex and its sides have no common points other than the vertices and bound a disk. By $\mathrm{AZ}$, Chapter 3, Theorem 3], $M$ can be partitioned into arbitrarily small simple triangles such that the strict triangle inequality is fulfilled for each of them. Moreover, any finite set of points can be included in the set of vertices in such a way that some initial intervals of a prescribed finite collection of minimizers starting at these points lie along edges. If we replace each triangle of the partition by its comparison triangle (i.e., a planar triangle with the same side lengths), we get a surface $P$ equipped with a polyhedral metric. In [AZ, Chapter 7, Theorem 7] is was proved that, as the triangles of the partitions become smaller, the sequence of polyhedra $P_{i}$ converges to $M$ uniformly and regularly.

To ensure Lipschitz convergence, we specify the partition described above. Namely, let $\theta_{0}=\frac{1}{100} \min _{p \in M}(2 \pi-\omega(p))$. There are only finitely many points, say, $E_{1}, \ldots, E_{m}$, whose absolute curvatures exceed $\theta_{0}$. We construct a partition such that the star of each point $E_{k}, k=1, \ldots, m$, consists of isosceles triangles with vertex $E_{k}$ and such that the angles of the triangles at $E_{k}$ are in the interval $\left(2 \theta_{0}, 10 \theta_{0}\right)$. Moreover, the triangles of this partition are so small that $\widetilde{\Omega}(T)<0,001 \theta_{0}$ for every triangle $T$. Since the curvature of the triangles is small, all the angles except, possibly, one angle in each triangle are less than $\pi-5 \theta_{0}$. Cutting each triangle with a "large" angle into two triangles, we get a partition such that all angles of the triangles are less than $\pi-5 \theta_{0}$.

Now we slightly change our partition to get a partition all angles of which are positive. For this, we replace some ordinary triangles by generalized ones. We can do this in such a way that the stars of the points $E_{k}$ remain unchanged and every modified side becomes a polygonal line with almost the same length and turn (see the details in $\mathrm{BeBu}$, Lemma 6]). This deformation is assumed to be so small that all the properties listed above are preserved.

Let $i$ be so large that $\frac{1}{i} \ll \theta_{0}$, and let $\delta_{i}=\delta\left(\theta_{0}, \frac{1}{i}\right)>0$ denote the number corresponding to $\theta_{0}$ and to $L_{i}=1+\frac{1}{i}$ in accordance with Lemma 3 . We can choose the partition of $M$ into generalized triangles $T$ so that, in addition to the properties mentioned above, the following is true: $\widetilde{\Omega}(T)<0.001 \min \left\{\delta_{i}, \theta_{0}\right\}$ and $\operatorname{diam} T<\frac{1}{i}$.

We denote by $M^{i}$ the surface $M$ equipped with the partition described above. Since all angles of the (generalized) triangles $T_{i j}$ of this partition are nonzero, these angles are not less than some number $\beta_{i}>0$. If all angles of a triangle $T_{i j}$ are at least $\theta_{0}$, then $T_{i j}$ is $L_{i}$-bi-Lipschitz-equivalent to its comparison triangle (Lemma 31). In particular, this is true for all triangles adjacent to the vertices $E_{k}$.

In any triangle $T_{i j}$, only one angle can be less than $\theta_{0}$ (because such a triangle has no "large" angles and its curvature is small). Suppose the angle $\angle A B C$ of the triangle $\triangle A B C$ is less than $\theta_{0}$; the other angles of $\triangle A B C$ exceed $\theta_{0}$. Obviously, such a triangle cannot be adjacent to one of the vertices $E_{k}$. We take points $A_{1}, C_{1}, B_{1}$ on the sides $A B$, $B C, A C$ so that $\left|A A_{1}\right|=\left|A B_{1}\right|,\left|C C_{1}\right|=\left|C B_{1}\right|,\left|B A_{1}\right|=\left|B C_{1}\right|$ ("Gromov's product") and connect these points to each other with minimizers in the induced metric of the triangle. Observe that, due to the smallness of $\widetilde{\Omega}(\triangle A B C)$, these minimizers cut $\triangle A B C$ 
into 4 simple (generalized) triangles all angles of which except, possibly, $\angle A_{1} B C_{1}$ exceed $\theta_{0}$. Now we choose points $A_{2} \in A_{1} B, C_{2} \in C_{1} B, B_{2} \in A_{1} C_{1}$ such that $\left|A_{1} A_{2}\right|=\left|A_{1} B_{2}\right|$, $\left|C_{1} C_{2}\right|=\left|C_{1} B_{2}\right|,\left|B A_{2}\right|=\left|B C_{2}\right|$ and continue this process. It is not difficult to show that, since the curvature is small, all angles of the triangles $\triangle A_{k} A_{k+1} B_{k+1}, \triangle C_{k} C_{k+1} B_{k+1}$, $\triangle B_{k} A_{k} C_{k}$ are bounded from below by $\theta_{0}$ (we set $A=A_{0}, C=C_{0}, k=0,1, \ldots$ ) and that the strict triangle inequality is fulfilled for the sides of these triangles. Hence, all these triangles are $L_{i}$-bi-Lipschitz-equivalent to their comparison triangles. It is easily seen that $A_{k} \rightarrow B, B_{k} \rightarrow B$ as $k \rightarrow \infty$. Therefore, there is a number $k$ such that $\widetilde{\Omega}\left(\triangle A_{k} B C_{k}\right)<\delta\left(\beta_{i}, L_{i}\right)$. This means that $\triangle A_{k} B C_{k}$ is $L_{i}$-bi-Lipschitz-equivalent to its comparison triangle (Lemma 3). Now, replacing each triangle of our partition of $\triangle A B C$ by its comparison triangle (and, of course, doing this for each triangle $\triangle A B C$ ), we obtain a polyhedron $P_{i}$ that is $L_{i}$-bi-Lipschitz-equivalent to $M$. Since the sides of the triangles are "almost straight", regular convergence is easily verified.

Lemma 6 is proved.

Lemma 7. For any $\nu>0$ and $d>0$, each compact (possibly, with boundary) Aleksandrov surface $M$ without peak points allows for a triangulation $\left\{T_{k}\right\}$ such that

(i) $\widetilde{\Omega}\left(T_{k}\right)<\nu$;

(ii) $\operatorname{diam} T_{k}<d$;

(iii) all angles of the triangles $T_{k}$ are at least $\alpha(\theta)>0$, where $\alpha$ depends only on $\theta=\min \left\{\min \left\{2 \pi-\omega^{+}(p): p \in M\right\}, \min \{\pi-\tau(q): q \in \partial M\}\right\}$, and $\tau(q)$ is the turn at the point q;

(iv) the set of vertices of the triangulation contains any prescribed finite set of points $E_{k} \in M$.

Remark 4. a) In the case of polyhedral metrics, this lemma was in fact proved in $\mathrm{B}$, Theorem 2] on the basis of a theorem from [BZ] (see also [BZ1]); all triangles of the triangulation are flat in this particular case.

b) It seems plausible that, by using Chebyshev coordinates, Lemma 7 can be proved in the same way as this was done for polyhedra in [B, BZ]. However, it is simpler to reduce Lemma 7 to the case of polyhedra with the help of Lemma 6 (this approach was suggested by A. Belen'kiı)).

Proof. By Lemma 6, $M$ can be Lipschitz-approximated by a sequence of polyhedra $P_{i}$. Let $f_{i}: P_{i} \rightarrow M$ be the corresponding $L_{i}$-bi-Lipschitz maps, $L_{i} \rightarrow 1$ as $i \rightarrow \infty$. We choose and fix a finite set $\left\{F_{k}\right\}$ on $M$; in particular, in this set we include all points with variation of curvature exceeding $\frac{1}{10} \nu$. Denote $F_{k i}=f_{i}^{-1}\left(F_{k}\right)$. As was mentioned in Remark 4a, each $P_{i}$ can be triangulated into planar triangles satisfying conditions (i)-(iv) of the lemma, even with the replacement of $\nu$ and $d$ by $\frac{1}{100} \nu$ and $\frac{1}{10} d$. Moreover, we incorporate all points $F_{k i}$ into the set of vertices of the required triangulation of $P_{i}$. Also, we suppose that the triangles are so small that every $d$-neighborhood of each point $A \in M$ contains at most one point $F_{k}$ and the absolute curvature of such a neighborhood with the point $F_{k}$ removed does not exceed $\frac{1}{20} \nu$. We may assume that the same is true for every polyhedron $P_{i}$ if $i$ is sufficiently large. This triangulation of $P_{i}$ can be chosen in such a way that all the angles of the triangles are bounded from below by some number $2 \alpha_{i}$ depending only on

$$
\theta_{i}=\min \left\{\min \left\{2 \pi-\omega^{+}(p): p \in P_{i}\right\}, \min \left\{\tau(q): q \in \partial P_{i}\right\}\right\} ;
$$

in particular, $2 \alpha_{i}$ does not depend on the smallness of the triangles. Observe that if $i$ is sufficiently large, then the numbers $\theta_{i}$ for the polyhedra $i$ are almost the same as the corresponding number $\theta$ for $M$. Let $\alpha=\theta / 2$. Now we use Lemma 3 Since $\alpha$ does not depend on the smallness of the triangles, on the choice of the points $\left(F_{k}\right)$, and on $\nu$ and 
$d$, we may assume $\nu$ to be so small (as compared to $\alpha$ ) that $2 \nu<\delta=\delta(\alpha, L=2$ ), where $\delta$ is as in Lemma 3 .

Now we fix $i$ and join by minimizers the points of $M$ whose inverse images in $P_{i}$ are joined by edges of the triangulation. We claim that if $i$ is sufficiently large, then the resulting triangulation of $M$ is combinatorially equivalent to the triangulation of $P_{i}$, all angles of this triangulation are bounded away from zero by a number depending only on $\theta$, and the angles at $F_{k}$ differ only slightly (by less than $2 \nu$ ) from the corresponding angles at $F_{k}$.

Indeed, let $A B$ and $B C$ be edges of the triangulation of $P_{i}$, and let $A^{\prime} B^{\prime}$ and $B^{\prime} C^{\prime}$ be the corresponding minimizers in $M$. The minimizers $A B$ and $B C$ split a neighborhood of $B$ into two sectors. The sector corresponding to the triangle $A B C$ is distinctly smaller, and the angle of this sector is equal to the angle $\angle A B C$ of the triangle. Moreover, if $i$ is large, then $\angle A^{\prime} B^{\prime} C^{\prime}$ is almost equal to $\angle A B C$. Now, the combinatorial equivalence of the nets in question follows easily. The other properties of the triangulation of $M$ follow from the corresponding properties of the triangulations of the polyhedra $P_{i}$ (for sufficiently large $i$ ).

Lemma 7 is proved.

\section{§5. Proof of the Key lemma}

1. Preliminary agreements. Here we shall consider only a sequence of surfaces $M_{j} \in$ $\mathfrak{M}$ converging (in the Gromov-Hausdorff topology) to a surface $M$. We shall construct partitions of these surfaces into triangles. These triangles are assumed to be so small that the values of the parameters $\chi, D, l$ occurring in the definition of the class $\mathfrak{M}$ do not play any role in our further considerations. By the bi-Lipschitz equivalence of triangles or more general figures we shall mean the existence of a bi-Lipschitz map with constant depending only on $C$ and $\epsilon$. If there are marked points at the boundary of a figure (the vertices of a triangle are always viewed as marked), then we assume that our map takes marked points to marked points and that the restriction of the map to any boundary curve connecting marked points is always linear.

2. Choice of scales. We deal with three scales. First, it is the size of the angles of the triangles. Partitions of the limit surface $M$ involve two types of triangles: "ordinary" and "special" ones. In accordance with Lemma 7 , the angles of the ordinary triangles

are bounded away from zero by a constant $\lambda>0$ depending only on $C$ and $\epsilon$. All special triangles are isosceles, and the angles at their vertices belong to some interval $\left(\varphi_{0}, \varphi_{1}\right)$. The numbers $\varphi_{i}$ are small and positive and also depend only on $C$ and $\epsilon$; they will be chosen in item 3 of the proof.

As the second step, we choose a small positive number $\delta$ so as to ensure that the conclusions of Lemmas 3 and 4 be valid even if the angles of the triangles are bounded from below by the number $0.01 \pi \varphi_{0}(2 \pi+C)^{-1}$ instead of $\varphi_{0}$. Some quantities dominated by $C^{*} \delta$ (as always, $C^{*}$ depends only on $C$ and $\epsilon$ ) will arise in the course of the proof. We denote such quantities by $\delta^{\prime}$. It is important that we can unboundedly reduce $\delta$ and, with it, $\delta^{\prime}$, keeping $C$ and $\epsilon$ fixed. For this reason we will keep the notation $\delta$ for all quantities of the form $m \delta$ if $m$ is not too large (say, $m<50$ ). It is convenient to assume that $\delta^{\prime} \ll \min \left\{\varphi_{0}, \lambda\right\}$.

After we have fixed $\varphi_{i}$ and $\delta$, we choose a partition of $M$ into triangles so small that the total curvature of every triangle be less than $\delta$ (by the total curvature of a triangle $T$ we mean $\widetilde{\Omega}(T)$ ). In fact, we deal with even finer partitions. This will help us to transfer a partition to the surfaces $M_{j}$ for large values of $j$. 
Finally, fixing a partition, we choose an integer $j_{0}$ so large that, for $j>j_{0}$, substantial portions of the curvature of $M_{j}$ are concentrated in very small (in comparison with the size of the triangles) neighborhoods of the vertices.

Let us explain this. K. Fukaya defined weak convergence of measures for the case of Gromov-Hausdorff convergence of spaces; see the details in [Sh]. For a subsequence, the curvatures $\omega_{j}$ of $M_{j}$ converge weakly to the curvature $\omega$ of $M$; the positive and negative parts $\omega_{j}^{+}, \omega_{j}^{-}$of $\omega$ converge weakly to some finite measures $\mu^{+}, \mu^{-}$. We have $\mu^{ \pm} \geq \omega^{ \pm}$, where the $\omega^{ \pm}$are the positive and negative parts of $\omega$. Choosing a partition of $M$ into triangles, we require that not only the total curvature of the triangles be small, but also the values of $\mu^{+}$and $\mu^{-}$on all triangles with vertices removed be small (less than $\delta$ ). (Note that both measures $\mu^{+}$and $\mu^{-}$can simultaneously take large values at a vertex. The reason is that the convergence $M_{j} \rightarrow M$ may fail to be regular.) All vertices for which these measures can be large will be included in the set of vertices of special triangles. For converging surfaces $M_{j}$, the measures $\omega_{j}^{ \pm}$are not necessarily concentrated at vertices; they may be "spread out". However, for any $R>0$ there exists a number $j_{0}$ such that, for each vertex $B$ of each special triangle $T$, almost all measure $\omega^{ \pm}(T)$ is concentrated in the $R$-neighborhood $V=\mathbf{D}(B, R)$ of the point $B_{j}$ for $j>j_{0}$.

This means that for every special triangle $\triangle A_{j} B_{j} C_{j}=T \subset M_{j}$ we have

$$
\omega_{j}^{ \pm}(T \backslash V)<\delta .
$$

In what follows, we assume that $j$ is so large that inequality (1) is fulfilled for $R$ chosen as above.

3. Special vertices and triangles. Now we use Lemma 7 to construct a special partition of the limit surface $M$. We choose a finite set of points $F_{k} \in M$ and triangulate small closed neighborhoods $Q_{k}^{0}$ of these points in a special way; in particular, $Q_{k}^{0} \cap Q_{l}^{0}=\varnothing$ for $k \neq l$. After that, we apply Lemma 7 to the surface $M_{0}=M \backslash \bigcup_{k} Q_{k}^{0}$ with boundary. As a result, we obtain a partition of $M$ into triangles.

As the neighborhoods $Q_{k}^{0}$ we take stars of the points $F_{k}$; these stars consist of isosceles triangles $\triangle F_{k} A_{k i} A_{k i+1}$, where $\left|F_{k} A_{k i}\right|=\left|F_{k} A_{k i+1}\right|$. We say that the points $F_{k}$ and the triangles $\triangle F_{k} A_{k i} A_{k i+1}$ adjacent to them are special. The construction of these stars allows some freedom; in particular, we can vary angles of special triangles, their size, and the fineness of triangulation. We use this freedom as follows.

Let $C$ and $\epsilon$ be the constants occurring in the definition of the class $\mathfrak{M}$, and let $C_{1}=2 \pi+C$. First (before fixing the points $F_{k}$ ), we choose intervals for the admissible values of the angles with vertices at $F_{k}$. These angles must be so small that even after multiplication by $2 \pi / \epsilon$ they remain "small", say, less than 0.001 . On the other hand, we must bound these angles uniformly from below and bound the number of edges at a special vertex from above. Thus, we require that the angles $\psi$ with vertices at $F_{k}$ be in the interval

$$
\varphi_{0}=10^{-5} \epsilon<\psi<\varphi_{1}=10^{-4} \epsilon .
$$

These conditions can always be ensured, and the number $m$ of the edges at $F_{k}$ is uniformly bounded from above:

$$
m<10^{6}(2 \pi+C) \epsilon^{-1}
$$

Now we choose a number $\delta$, which characterizes the smallness of the curvature of the triangles. Namely, we take $L=\frac{11}{10}$, and let $\delta_{1}$ correspond to the numbers $L$ and $\alpha=\lambda$ in accordance with Lemma 3 Similarly, we can find $\delta_{2}$ corresponding to the numbers $L$ and $0.01 \varphi_{0} C_{1}^{-1}$. Then Lemma 4 gives us $\delta_{3}$ corresponding to $\phi=0.1 \varphi_{0}, \phi_{1}=0.01$. Finally, we put $\delta=\frac{1}{100} \min _{i}\left\{\delta_{i}\right\}$. Thus, $\delta$ depends only on $C$ and $\epsilon$. Recall that, if necessary, we 
can reduce $\delta$ and then find a partition of $M$ into triangles whose absolute curvatures do not exceed the new value of $\delta$; this does not influence the lower bounds for the angles of the triangles. Since $\varphi_{1}<0.01$, we may assume that the angles adjacent to the bases of special triangles are close to $\frac{\pi}{2}$ (with accuracy $\varphi_{0}$ ).

After we fix the set $\left\{F_{k}\right\}$ (this will be done shortly), we choose the stars $Q_{k}^{0}$ of these vertices to be so small that $\Omega\left(Q_{k}^{0} \backslash F_{k}\right)<\delta$ and $\operatorname{diam} Q_{k}^{0}<\delta$. Then each special triangle will be $\frac{11}{10}$-bi-Lipschitz-equivalent to its comparison triangle. Observe that the turn of the boundary of $Q_{k}^{0}$ from outside at each point is not large, say, less than $\frac{\pi}{2}$.

4. Partition of $M$ into triangles. We triangulate the surface $M^{\prime}=M \backslash \bigcup_{k} Q_{k}^{0}$ in accordance with Lemma 7 . All angles of such a triangulation are bounded from below by some $\lambda>0$, which depends on the number $\theta$ for $M_{0}$ (see item (iii) of Lemma 7). Actually, this $\theta$ does not depend on our choice of the vertices $F_{k}$ and their stars, so that we can take $\theta=\epsilon$. Indeed, as was mentioned before, the outside turn of the boundary of any star at any point does not exceed $\frac{1}{2} \pi$. At the same time, including all points with large values of $\mu^{ \pm}$in the set $\left\{F_{k}\right\}$ and taking a sufficiently fine triangulation, we can guarantee the inequality $\widetilde{\Omega}(T)<\delta$ for all triangles, where $\delta$ is as above.

Thus, from the outset we include all the points having curvature $\Omega\left(F_{k}\right) \geq \delta$ in the set $\left\{F_{k}\right\}$; after that we choose the stars $Q_{k}$ to be so small that $\Omega\left(Q_{k} \backslash F_{k}\right) \leq \bar{\delta}$, and finally, we triangulate $M_{0}$ so that the inequality $\widetilde{\Omega} T \leq \delta$ be fulfilled for all triangles. This is possible because our constants do not depend on the choice of the set of points $F_{k}$, the stars $Q_{k}$, and a triangulation. In fact, at the beginning of item 5 of the proof we shall add some requirements (which can easily be ensured) on the choice of the partition for $M$.

As a result, we get a partition of $M$ into two kinds of triangles: special ones and others, and each triangle $T$ satisfies $\widetilde{\Omega}(T)<\delta$ and is $\frac{11}{10}$-bi-Lipschitz-equivalent to its comparison triangle.

5. Converging surfaces. Lemma 6 allows us to assume that the converging surfaces $M_{j}$ are equipped with polyhedral metrics. Taking a subsequence, we may also assume that the curvatures $\omega_{j}$ of the surfaces $M_{j}$ converge weakly (in the sense of the definition given in [Sh]) to the curvature of $M$, and that their positive and negative parts $\omega_{j}^{+}, \omega_{j}^{-}$ converge weakly to some finite measures $\mu^{+}, \mu^{-}$. Recall that $\mu^{ \pm} \geq \omega^{ \pm}$, where the $\omega^{ \pm}$ are the positive and the negative part of the curvature of $M$.

Consider a partition of $M$ chosen in accordance with item 4 of the proof. Let $\left\{A_{i}\right\}$ be the set of all vertices of that partition, and let $\left\{F_{k}\right\}$ be the subset of all special vertices. Replacing this set by a wider set $\left\{F_{k}\right\}$, choosing smaller stars $Q_{k}$, and making triangles smaller, we could include in $\left\{F_{k}\right\}$ all points $X \in M$ such that $\mu^{ \pm}(X) \geq \delta$. In the same way, we can ensure that every closed triangle with removed vertices satisfies the inequality $\mu^{ \pm}<\delta$. Condition (iii) in the definition of the class $\mathfrak{M}$ implies that $\mu^{+}(X) \leq 2 \pi-\epsilon$ for every $X \in M$. As a result, we can take all triangles to be so small that the inequality $\mu^{+}\left(Q_{k}^{0}\right)<2 \pi-\frac{2}{3} \epsilon$ is fulfilled for each star.

We require that the diameters of all triangles do not exceed a small number $d>0$; this number is chosen so as to ensure the following:

(a) the inequality $\mu^{ \pm}(E)<\delta$ is fulfilled for every set $E$ that contains no points $F_{k}$ and $\operatorname{satisfies} \operatorname{diam}(E) \leq 10 d$;

(b) each circle of radius $10 d$ contains at most one vertex $F_{k}$.

Let $A_{j k}$ be points of the surface $M_{j}$ such that $A_{j k} \underset{\mathrm{GH}}{\longrightarrow} A_{k}$ as $j \rightarrow \infty$; in particular, $F_{j k} \underset{\mathrm{GH}}{\longrightarrow} F_{k}$. If a vertex $A_{k}$ belongs to the boundary of a star $Q_{l}^{0}$, then we choose points $A_{j k}$ so that $\left|F_{j l} A_{j k}\right|=\left|F_{l} A_{k}\right|$. 
Next, we assume that the numbers $j$ are so large that if a set $B \subset M_{j}$ has diameter not exceeding $6 d$ and does not intersect the $\delta$-neighborhoods of the points $F_{j k}$, then $\Omega_{j}(B)=\omega_{j}^{+}(B)+\omega_{j}^{-}(B)<\delta$.

6. Partitions of $M_{j}$ and nonspecial triangles. To construct a partition of the surface $M_{j}$, we connect a pair of points $A_{j k}$ by a minimizer if and only if the corresponding pair of points $A_{k}$ is connected by a minimizer. Such minimizers are not necessarily unique and a priori may have superfluous intersections. We choose these minimizers so as to avoid such unnecessary intersections. Note that the minimizers connecting $A_{j k}$ with $A_{j s}$ may fail to converge (in the Gromov-Hausdorff topology) to the minimizer chosen beforehand and connecting $A_{k}$ with $A_{s}$. Nevertheless, almost the same arguments as in Lemma 7 show that we get a partition of $M_{j}$ that is combinatorially equivalent to the partition of $M$.

Let $\triangle A B C$ be a nonspecial triangle on the surface $M$. Its angles are almost the same as the angles of its comparison triangle. If the numbers $j$ are sufficiently large, then the triangles $\triangle A_{j} B_{j} C_{j}$ are located in regions with small (less than $\delta$ ) variation of curvature. Hence, the angles of such a triangle are almost equal to the angles of its comparison triangle. By Lemma 4 in $\left[\mathrm{BeBu}\right.$, both triangles $\triangle A B C$ and $\triangle A_{j} B_{j} C_{j}$ are bi-Lipschitz-equivalent to their comparison triangles with a constant $L$ depending only on $\lambda$ and $\delta$ (in the notation of that lemma). If $\delta$ is sufficiently small, then this constant can be chosen as close to 1 as we wish. For large $j$, the comparison triangles, $\triangle A B C$ and $\triangle A_{j} D_{j} C_{j}$, are almost equal. Therefore, all nonspecial triangles of the surfaces $M_{j}$ are bi-Lipschitz-equivalent to the corresponding triangles of the surface $M$.

Now, to finish the proof, it suffices to verify that, for large $j$, every special triangle of the surface $M_{j}$ is bi-Lipschitz-equivalent to the corresponding triangle of the surface $M$ or, in other words, to its comparison triangle.

7. Special triangles. Let $Q_{0}$ be the star of a fixed vertex $B^{0}=E_{k}$ of the surface $M$, and let $Q$ be the star of the corresponding vertex $B=E_{k j}$ of the surface $M_{j}$. Recall that every triangle of $Q_{0}$ is almost flat; hence, it is bi-Lipschitz-equivalent to its comparison triangle. The latter is bi-Lipschitz-equivalent to a comparison triangle for the corresponding triangle of $Q$. Therefore, it suffices to prove that, for sufficiently large $j$, every star $Q$ is bi-Lipschitz-equivalent to the star glued from comparison triangles for the triangles of $Q$.

8. Plan of the further proof. We are going to apply arguments used in $\mathrm{BL}$ to $Q 2$ For this, we attach an appropriate locally flat surface to $Q$ so as to obtain a complete surface $P$ homeomorphic to the plane and having the same positive and negative curvature as $Q$. It is easily seen that this is possible.

Roughly speaking, the key ingredient of the proof in $\mathrm{BL}$ is as follows. If $P$ is a polyhedral surface homeomorphic to the plane, and $\omega^{+}(P) \leq 2 \pi-\epsilon<2 \pi$ and $\omega^{-}(P) \leq$ $C<\infty$, then on $P$ there exists a finite set of flat sectors with disjoint interiors and such that every point of nonzero curvature is a vertex of some of these sectors. Depending on the curvature sign, we can reduce or enlarge the angles of these sectors so as to eliminate the curvature at the sector vertices. The size of the sector angles can be chosen in such a way that these deformations give a bi-Lipschitz map with constant $L$ depending only on $C$ and $\epsilon$. This yields a bi-Lipschitz map of $P$ to the plane $\mathbb{R}^{2}$.

In fact, the situation in $[\mathrm{BL}$ is more intricate: the transformations mentioned above are fulfilled in three steps. First, $P$ is divided into two half-planes by a special quasigeodesic line, and we look for flat sectors separately in each half-plane. At the second

\footnotetext{
${ }^{2}$ See footnote 1 on page 945.
} 
step, sectors with vertices at points of positive curvature are found, and the curvature at these points is eliminated with the help of stretching these sectors. At the third step, a similar process allows us to eliminate all negative curvature; see the details in [BL].

In our situation, there is an obstruction to direct application of this construction to $P$ : it may happen that some of the flat sectors we have found contain rays forming very small angles with the boundary $\Gamma=\partial Q$ of the star $Q$. Only sectors with vertices close to $\Gamma$ can have this property. To avoid this, we choose $j_{0}$ so large that, for $j>j_{0}$, the curvature of $Q$ is concentrated almost entirely in a small neighborhood $V$ of the central point $B \in Q$. After that, we replace a relatively wide collar of $\partial Q$ in $Q$ by a flat collar. As a result, the flat sectors turn out to be "almost orthogonal" to $\partial Q$ on the modified surface. This simplifies our further considerations.

9. Elimination of curvature near $\partial Q$. Let $\left\{A_{i}\right\}$ be the set of vertices of $\partial Q$, and let $\left|B A_{i}\right|=R_{0}$. We put

$$
\kappa=10 \max \left\{\frac{2 \pi}{2 \pi-\epsilon}, \frac{2 \pi+C}{2 \pi}\right\} .
$$

Consider the disk $\mathbf{D}(B, R)$ of radius $R$ such that

$$
10 \kappa R<\delta R_{0}
$$

Next, we choose a disk $\mathbf{D}(B, r)$ (where $r \ll R$ ) and a large number $j_{0}$ so as to have $\mu^{ \pm}(Q \backslash \mathbf{D}(B, r))<\delta$ for $j>j_{0}$, and we assume that the conditions of Lemma 5 are fulfilled for $\Psi=C, Z=B$.

We are going to show that every star $Q$ is bi-Lipschitz-equivalent to a region $Q^{\prime}$ (equipped with a polyhedral metric) that is flat everywhere except for a small $C^{*} r$ neighborhood of a point $Z^{\prime}$ located at a distance $C^{*} R_{0}$ from the boundary of $Q^{\prime}$, and, moreover, $\mu^{-}\left(Q^{\prime}\right)<C+\delta$ and $\mu^{+}\left(Q^{\prime}\right)<2 \pi-\frac{1}{2} \epsilon$.

To simplify notation, we omit indices and denote the triangle $\triangle B A_{i} A_{i+1}$ by $\triangle B A C$. We pick points $A_{1}, C_{1}$ on the minimizers $B A, B C$ at a distance $r / 2$ from $B$ and connect these points by a minimizer $A_{1} C_{1}$.

We refine our requirement concerning $j_{0}$, namely, we choose $\rho>0$ so small and $j_{0}$ so large that the conditions of Lemma 5 be fulfilled even if we replace $R$ and $r$ with $r$ and $\rho$, respectively. In particular, we have $\mu^{ \pm}(Q \backslash \mathbf{D}(B, \rho))<\delta$. It is not difficult to see that in this case $A_{1} C_{1}$ lies in $\mathbf{D}(B, r)$ and does not meet the disk $\mathbf{D}(B, r / 4)$. Moreover, the angles $\angle B A_{1} C_{1}$ and $\angle B C_{1} A_{1}$ are "almost equal" to the angles $\angle A_{1}^{\prime}$ and $\angle C_{1}^{\prime}$ of the comparison triangle $\triangle B^{\prime} A_{1}^{\prime} C_{1}^{\prime}$ (i.e., their differences do not exceed $\delta$ ). In particular, these angles are less than $\frac{1}{2}\left(\pi-\varphi_{0}\right)$. It is easily seen that the conditions of Corollary 2 are fulfilled for the quadrangle $A A_{1} C_{1} C$ (with an appropriate $\phi$ ). Application of this corollary allows us to replace each triangle $\triangle B A_{i} A_{i+1}$ by a triangle that is flat off the disk $\mathbf{D}(B, r)$ and is $L$-bi-Lipschitz-equivalent to $\triangle B A_{i} A_{i+1}$. As a result of this replacement, the total curvature of the triangle could increase (at the points $A_{1}, C_{1}$ ), but the change of curvature does not exceed $C^{*} \delta$. Choosing $\delta$ sufficiently small and $j_{0}$ sufficiently large, we can make the constant $L$ be as close to 1 as we wish.

We keep the old notation $Q, B, A_{1} A_{2} \ldots A_{m}$ for the star formed by the new triangles and for the elements of this star.

Also, we assume that $r$ is so small in comparison with $R$ that $\triangle A B C$ satisfies the conditions $A \in \mathbf{D}(B, \kappa r),|B C| \geq \kappa^{-1} R$.

10. Flat sectors. We want to prove that the new star $Q$ is bi-Lipschitz-equivalent to the star obtained by gluing together comparison triangles for the triangles of $Q$. For this, we use the construction employed in $\mathrm{BL}$ and described briefly in item 8. As has already been mentioned, this construction will be applied twice: first, to eliminate the positive 
curvature and, after that, to exclude the negative curvature. Since these two steps are similar, we describe only the first of them in detail.

We add a flat annulus to $Q$ to obtain an open complete surface $P$ that is flat everywhere except the disk $\mathbf{D}(B, r) \subset Q$. This is possible. Indeed, denote by $\alpha_{i}^{-}$and $\alpha_{i}^{+}$the angles of $\triangle A_{i} B A_{i+1}$ adjacent to the base. Consider a flat region bounded by two rays and an interval of length $\left|A_{i} A_{i+1}\right|$; we assume that the angles between the interval and the rays from the side of the region are equal to $\pi-\alpha_{i}^{-}, \pi-\alpha_{i}^{+}$, respectively. To get $P$, we glue such flat regions together along rays and attach the resulting annulus to $Q$. For $j$ sufficiently large, the positive curvature of $P$ is less than $2 \pi-\epsilon-\delta=2 \pi-\epsilon^{\prime}$ and its negative curvature is less than $C+\delta=C^{\prime}$. Since our estimates are rough, we preserve the previous notation $\epsilon$ and $C$ for $\epsilon^{\prime}$ and $C^{\prime}$.

In accordance with [BL], on $P$ there exists a finite collection of flat sectors with disjoint interiors and such that all vertices of positive curvature occur among vertices of these sectors, and the sum of angles for the sectors with common vertex $O$ is equal to

$$
\frac{2 \pi-\omega^{+}(P)}{\omega^{+}(P)} \omega^{+}(O) \text {. }
$$

To eliminate the positive curvature at the point $O$, we stretch all sectors with vertex $O$ by multiplying their angles by $L_{1}=\frac{2 \pi}{2 \pi-\omega^{+}(P)}$. As a result, we obtain a polyhedron $P_{1}$ of nonpositive curvature and bi-Lipschitz-equivalent to $P$.

After this step, we find a similar system of flat sectors with vertices at the points of negative curvature, and then eliminate the negative curvature in the same way by means of compressing the flat sectors of $P_{1}$. Finally, we get a bi-Lipschitz map $f: P_{1} \rightarrow \mathbb{R}^{2}$ with the Lipschitz constant $\left(\frac{2 \pi+C}{\epsilon}\right)^{\frac{1}{2}}$.

Following [BL, we use maps of the form $(r, \phi) \rightarrow(r, a \phi)$ for stretching and compressing sectors, where $(r, \phi)$ are polar coordinates with origin at the vertex of the sector. We may assume that the angles of the sectors are small; in particular, that each sector intersects the base of only one special triangle, and that the central point $B$ of the star $Q$ does not belong to the interior of any sector. To achieve this, it suffices to split sectors into smaller ones.

Actually, we consider not the entire surface $P$, but only the star $Q$ of the point $B$. Such a star consists of the isosceles triangles $\triangle A_{i} B_{j} A_{i+i}$ and is bounded by the polygonal line $\Gamma=A_{1} A_{2} \ldots A_{m}$. From the description of the map $f$, it is clear that $Q$ is bi-Lipschitzequivalent to the flat region $\widetilde{Q}=f(Q)$. The map $f$ takes the bases $\Gamma_{i}=A_{i} A_{i+1}$ of the triangles $\triangle A_{i} B_{j} A_{i+i}$ to curves $\widetilde{\Gamma}_{i}$ (nonsmooth in general). These curves consist of straight segments (the images of the segments that do not belong to a flat sector) and smooth curves (the images of the intersections of $\Gamma_{i}$ with flat sectors). (It is not important for us what the images of the lateral sides of the triangles $\triangle A_{i} B_{j} A_{i+i}$ look like.)

11. The flat region $\widetilde{Q}$. We are going to show that the flat region $\widetilde{Q}$ is bi-Lipschitzequivalent to a polygon glued from comparison triangles for the curved triangles forming $Q$.

We connect $\widetilde{B}$ with all points $\widetilde{A}_{i}$ by minimizers in the intrinsic metric of $\widetilde{Q}$ (avoiding unnecessary intersections). Thus, we split $\widetilde{Q}$ into "curved triangles" $\widetilde{T}_{i}$ with the curves $\tilde{\Gamma}_{i}$ as bases. (It will be clear in the sequel that these minimizers are almost orthogonal to $\widetilde{Q}$ and do not touch one another.)

It suffices to verify that

(a) each curved triangle $\widetilde{T}_{i}$ is bi-Lipschitz-equivalent to its comparison triangle (i.e., to a flat triangle $\widetilde{T}_{i}^{\prime}$ whose side lengths are equal to $\left|\widetilde{B} \widetilde{A}_{i}\right|,\left|\widetilde{B} \widetilde{A}_{i+1}\right|$, and $s\left(\widetilde{\Gamma}_{i}\right)$, respectively); 
(b) the flat triangle $\widetilde{T}_{i}^{\prime}$ is bi-Lipschitz-equivalent to a comparison triangle for $\triangle B A_{i} A_{i+1}$.

Since the latter triangle is almost equal (for large $j$ ) to the corresponding triangle of the star $Q^{0}$, this finishes the proof.

To prove (a) and (b), we need to estimate angle and distance distortions for the map $f$. To simplify exposition, we consider only one step (eliminating the positive curvature); for the second step (eliminating the negative curvature) estimates are similar.

12. Estimates. To prove (a), we use Lemma 4. The following statements show that $\widetilde{T}_{i}$ satisfies the conditions of that lemma. Also, they will help us to prove (b).

(i) For $j$ sufficiently large, the map $f$ only slightly changes the distances from $B$ to the boundary $\Gamma$ of $Q$. More precisely, for every $X \in \partial Q$ we have

$$
|| \widetilde{B} \widetilde{X}|-| B X||<C^{*} \delta|B X| .
$$

(ii) For $X \in \tilde{\Gamma}_{i}$, the angles between the radial minimizers $\widetilde{B} \widetilde{X}$ and the $\operatorname{arcs}$ of $\tilde{\Gamma}_{i}$ starting at $X$ are close to $\frac{1}{2} \pi$. In particular, the turns of $\tilde{\Gamma}_{i}$ at its angular points are small. The words "close" and "small" mean that the difference between the angles in question and $\frac{\pi}{2}$ does not exceed $\frac{1}{10}$.

The proof of (i). Let $X \in \partial Q$. We prove that $|B X|<(1+\delta)|\widetilde{B} \widetilde{X}|$. The second inequality is proved similarly.

Consider a minimizer $\tilde{\alpha}$ connecting $\widetilde{B}$ with $\widetilde{X} \in \partial \widetilde{Q}$, and let $\alpha$ be the $f$-inverse image of $\tilde{\alpha}$. By (5), the length of the initial arc $\tilde{\alpha}_{0}$ of $\tilde{\alpha}$ that goes from $\widetilde{B}$ to the boundary of the $f$-image of $\mathbf{D}(B, R)$ does not exceed $\kappa R<\frac{1}{10} \delta R_{0} \leq \frac{1}{5} \delta|B X|$.

If a segment of the minimizer $\alpha$ does not visit flat sectors, then the map $f$ does not change its length. If a segment of $\alpha$ goes off the disk $\mathbf{D}\left(\widetilde{B}, 2 r_{2}\right)$ and is contained in a flat sector we constructed, then it can only become shorter after application of $f$.

Now, let $\tilde{\beta}$ be an interval of $\tilde{\alpha}$ contained in a flat sector $\widetilde{S}$ obtained from $S$ by stretching, and let $\beta$ be the $f$-inverse image of the minimizer $\tilde{\beta}$ in $S$. Let $O$ and $\widetilde{O}$ be the vertices of the sectors $S$ and $\widetilde{S}$, respectively.

We denote by $Y$ and $Z$ the initial and the terminal point of the segment $\beta$, and by $\widetilde{Y}$ and $\widetilde{Z}$ the initial and the terminal point of $\tilde{\beta}$. If $Z$ belongs to $\partial Q$, we replace the sectors $S, \widetilde{S}$ by their subsectors $Z O Y, \widetilde{Z} \widetilde{O} \widetilde{Y}$, and preserve the notation $S, \widetilde{S}$ for the new sectors.

We can assume that $\tilde{\beta}$ does not intersect the initial segment $\tilde{\alpha}_{0}$; then the distance between $\widetilde{B}$ and $\widetilde{Y}$ is at least $R$, whence $\angle \widetilde{O} \widetilde{Y} \widetilde{B} \leq 3 \delta$ (see Lemma 5 (ii) and Remark 4 ).

We show that

$$
s(\tilde{\beta}) \leq\left(1+C^{*} \delta\right) s(\beta)
$$

(as usual, $C^{*}$ means a constant depending only on $C$ and $\epsilon$ ).

Put $\angle Y O Z=\phi, \angle \widetilde{Y} \widetilde{O} \widetilde{Z}=\tilde{\phi},|O Z|=|\widetilde{O} \widetilde{Z}|=b,|Y Z|=c,|\widetilde{Y} \widetilde{Z}|=\tilde{c}$, and $\pi-\angle O Y Z=$ $\chi$.

We place the triangles $\triangle O Y Z$ and $\triangle \widetilde{S} \widetilde{Y} \widetilde{Z}$ in $\mathbb{R}^{2}$ in one and the same half-plane with respect to their common side $O Y=\widetilde{O} \widetilde{Y}$. It is clear that $|\tilde{c}-c| \leq|\widetilde{Z} Z|=2 \sin \frac{1}{2}(\tilde{\phi}-\phi) b \leq$ $(\kappa-1) \delta c$, because $\phi<\chi \leq \delta$. The latter inequality follows from our choice of the disk $\mathbf{D}(B, r)$ at the beginning of item 9 and from Lemma 5 Estimate (7) is proved.

To obtain the second estimate, it suffices to take the minimizer $B X$ and its $f$-image for the roles of $\alpha$ and $\tilde{\alpha}$, respectively. 
The proof of (ii). We turn to the region $Q$ and, for brevity, denote $A_{i}=A, A_{i+1}=C$. The side $A C$ is small as compared to $|A B|=|C B|$ (see inequalities (21)). Therefore, the angles of the comparison triangle $\triangle A^{\prime} B^{\prime} C^{\prime}$ that are adjacent to the base $A^{\prime} C^{\prime}$ are close to $\frac{\pi}{2}$, and the angle $\angle A^{\prime} B^{\prime} C^{\prime}$ is small. By Lemma 5 (i), the angles $\angle B A C$ and $\angle B C A$ are also close to $\frac{\pi}{2}$.

Consider a triangle $\triangle B A X$, where $X \in A C$. Let $\triangle B^{\prime} A^{\prime} X^{\prime}$ be its comparison triangle. Again by Lemma $5(\mathrm{i})$, the angles $\angle A$ and $\angle X$ are equal to the angles $\angle A^{\prime}$ and $\angle X^{\prime}$, respectively. Since $\angle A^{\prime}$ is almost equal to $\frac{\pi}{2}, \angle A$ is also close to $\frac{\pi}{2}$. ("Close" means that their difference is dominated by $0.01 \epsilon+\delta$.) Since the angle $\angle A^{\prime} B^{\prime} X^{\prime}$ is small, it follows that the angle $\angle A^{\prime} X^{\prime} B^{\prime}$ is close to $\frac{\pi}{2}$. Now, again by Lemma 5 (i), the angle $\angle A X B$ is also close to $\frac{\pi}{2}$, and the same is true for the angle $\angle C X B$.

Let $S$ be a flat sector with vertex $O$, and let its sides intersect $A C$ at points $X, Y$. The point $O$ lies in the small neighborhood $\mathbf{D}(B, r)$ of $B$, but not necessarily in the triangle $\triangle A B C$. Lemma 5 (ii) shows that the angles $\angle O X B, \angle O Y B$ are small; therefore, the angles $\angle O X Y, \angle O Y X$ are close to $\frac{\pi}{2}$ (in the same scale: their difference is dominated by $0.01 \epsilon+\delta)$.

Now we pass to the sector $\widetilde{S}$ that is the image of $S$. All radii of the sector $S$ are almost orthogonal to $\Gamma_{i}$. A straightforward calculation shows that then all radii of the flat sector $\widetilde{S}$ are almost orthogonal to $\tilde{\Gamma}_{i}$. The difference between the latter angles and $\frac{\pi}{2}$ depends only on $\kappa$ and on the difference between $\frac{\pi}{2}$ and the angles that the radii of the flat sectors $S$ form with $\Gamma_{i}$; ultimately, the difference in question is dominated by a quantity depending only on $C$ and $\epsilon$. The vertices $O$ of the flat sectors $S$ are very close to the point $B$. Since $f$ multiplies distances by at most $\kappa$, the $f$-images of the vertices $O$ are close to $\widetilde{B}$. Consequently, the angles $\angle O X B$, where $X \in \Gamma_{i}$, are close to zero, so that all angles between the segments $\widetilde{B} \widetilde{X}$ (which are minimizers in $\widetilde{Q}$ ) and $\Gamma_{i}$ are close to $\frac{\pi}{2}$. (In particular, the flat region $\widetilde{Q}$ is starlike with respect to $\widetilde{B}$.) This proves (ii).

Estimate (i) implies that the differences between the lengths of the sides $\widetilde{A}^{\prime} \widetilde{B}^{\prime}, \widetilde{C}^{\prime} \widetilde{B}^{\prime}$ of the comparison triangle $\triangle \widetilde{A}^{\prime} \widetilde{B}^{\prime} \widetilde{C}^{\prime}$ and the lengths of the corresponding sides of the comparison triangle $\triangle A^{\prime} B^{\prime} C^{\prime}$ are small. By (ii), the ratio $\left|\widetilde{A}^{\prime} \widetilde{C}^{\prime}\right| /\left|\widetilde{A}^{\prime} \widetilde{C}^{\prime}\right|$ is bounded from below and from above by numbers depending only on $C$ and $\epsilon$. The choice of $\varphi$ and statement (ii) imply that the angle $\angle \widetilde{A}^{\prime} \widetilde{B}^{\prime} \widetilde{C}^{\prime}$ is less than $\frac{\pi}{2}$. Thus, it becomes clear that the flat triangles $\triangle \widetilde{A}^{\prime} \widetilde{B}^{\prime} \widetilde{C}^{\prime}$ and $\triangle A^{\prime} B^{\prime} C^{\prime}$ are $L$-bi-Lipschitz-equivalent, where $L$ depends only on $C$ and $\epsilon$ (see, e.g., [BeBu, Corollary 1]). Finally, each triangle $\triangle \widetilde{A} \widetilde{B} \widetilde{C}$ is bi-Lipschitz-equivalent to the corresponding triangle of the star $Q^{0}$, and our theorem is proved completely.

\section{REFERENCES}

[AZ] A. D. Aleksandrov and V. A. Zalgaller, Two-dimensional manifolds of bounded curvature, Trudy Mat. Inst. Steklov. 63 (1962), 262 pp.; English transl., Intrinsic geometry of surfaces, Transl. Math. Monogr., vol. 15, Amer. Math. Soc., Providence, RI, 1967, 327 pp. MR0151930|(27:1911) MR0216434(35:7267)

[Bak] I. Ya. Bakel'man, Chebyshev networks in manifolds of bounded curvature, Trudy Mat. Inst. Steklov. 76 (1965), 124-129; English transl. in Proc. Steklov Inst. Math. 1967. MR0209988 $(35: 883)$

[BeBu] A. Belen'kiŭ and Yu. Burago, Bi-Lipschitz-equivalent surfaces. I, Algebra i Analiz 16 (2004), no. 4, 24-40; English transl. in St. Petersburg Math. J. 16 (2005), no. 4. MR2090849

[BL] M. Bonk and U. Lang, Bi-Lipschitz parametrization of surfaces, Math. Ann. 327 (2003), 135169; (DOI: 10.1007/s00208-003-0443-8). MR2006006 (2004i:53100)

[B] Yu. Burago, Isometric imbedding of a manifold of bounded curvature into Euclidean space, Leningrad. Gos. Ped. Inst. Uchen. Zap. 395 (1970), 48-86. (Russian) MR0303473 (46:2610)

[BZ] Yu. Burago and V. Zalgaller, Polyhedral embedding of a net, Vestnik Leningrad. Univ. Mat. Mekh. Astronom. 1960, vyp. 2, 66-80. (Russian) MR0116317 (22:7112) 
[BZ1] - Isometric piecewise linear immersions of two-dimensional manifolds with polyhedral metrics into $\mathbb{R}^{3}$, Algebra i Analiz 7 (1995), no. 3, 76-95; English transl., St. Petersburg Math. J. 7 (1996), no. 3, 369-385. MR1353490 (96g:53091)

[I] S. V. Ivanov, Gromov-Hausdorff convergence and volumes of manifolds, Algebra i Analiz 9 (1997), no. 5, 65-83; English transl., St. Petersburg Math. J. 9 (1998), no. 5, 945-959. MR.1604389 (98k:53052)

[P] P. Petersen, A finiteness theorem for metric spaces, J. Differential Geom. 31 (1990), 387-395. MR.1037407 (91d:53070)

[Resh] Yu. G. Reshetnyak, Two-dimensional manifolds of bounded curvature, Geometry, 4, Itogi Nauki i Tekhniki Ser. Sovrem. Probl. Mat. Fund. Naprav., vol. 70, VINITI, Moscow, 1989, pp. 7-189; English transl., Encyclopaedia Math. Sci., vol. 70, Springer, Berlin, 1993, pp. 3-163. MR1099202 (92h:53104), MR:1263964

[Resh1] , Investigation of manifolds of bounded curvature in terms of isothermic coordinates, Izv. Sibirsk. Otdel. Akad. Nauk SSSR 1959, no. 10, 15-28. (Russian)

[Sh] T. Shioya, The limit spaces of two-dimensional manifolds with uniformly bounded integral curvature, Trans. Amer. Math. Soc. 351 (1999), 1765-1801. MR1458311(99h:53052)

St. Petersburg Branch, Steklov Mathematical Institute, Russian Academy of Sciences, Fontanka 27, St. Petersburg 191023, Russia

E-mail address: yuburago@pdmi.ras.ru

Received 16/MAR/2004

Translated by THE AUTHOR 